\title{
Seed-skewness algorithm for $x$-ray diffraction signal detection in the time-resolved synchrotron Laue photocrystallography
}

\author{
D. K. Szarejko, R. Kamiński, P. Łaski, K. N. Jarzembska \\ Department of Chemistry, University of Warsaw, Żwirki i Wigury 101, 02-089 Warsaw, Poland \\ katarzyna.jarzembska@gmail.com
}

Efficient 1-dimensional seed-skewness algorithm adapted for X-ray diffraction signal detection together with signal integration procedure are presented. The method was shown to work well for both the standard single-crystal X-ray diffraction data, as well as, for more specific photocrystallographic time-resolved Laue data collected at Advanced Photon Source and European Synchrotron Radiation Facility. It enables reasonable separation of signal from the background in single 1-dimentional data vectors, it is capable of determining small changes of reflection shapes and intensities resulting from exposure of the sample to laser light, and allows for extracting relatively weak reflections from the background. The last is possible through adjusting of "trust level" and "signal level" parameters in the algorithm. Otherwise, the procedure is objective and does relay only on skewness computation and its subsequent minimization, which enable the best possible background estimation. The intensities of strong reflections are determined comparably as via the Kruskal-Wallis test method, whereas weak reflections are more sensitive to the algorithm setting parameters. In turn, both methods estimate the background level equally-well.

Keywords: seed skewness; signal detection; integration; laue crystallography

R.K., D.S. and P.E. would like to thank the SONATA grant (2016/21/D/ST4/03753) of the National Science Centre in Poland for financial support. The time-resolved X-ray diffraction experiments were performed at the ID09 beamline of the European Synchrotron Radiation Facility (ESRF), Grenoble, France. The research used resources of the Advanced Photon Source, a U.S. Department of Energy (DOE) Office of Science User Facility operated for the DOE Office of Science by Argonne National Laboratory under contract No. DE-AC02-06CH11357. Use of BioCARS was also supported by the National Institute of General Medical Sciences of the National Institutes of Health (NIH) under grant No. R24GM111072 (note: the content is solely the responsibility of the authors and does not necessarily represent the official views of NIH). Time-resolved set-up at Sector 14 was funded in part through collaboration with Philip Anfinrud (NIH/NIDDK). 